\begin{tabular}{c} 
Ra Ximhai \\
$\begin{array}{c}\text { Revista de Sociedad, Cultura y } \\
\text { Desarrollo Sustentable }\end{array}$ \\
\hline
\end{tabular}

\author{
Ra Ximhai \\ Universidad Autónoma Indígena de México \\ ISSN: 1665-0441 \\ México
}

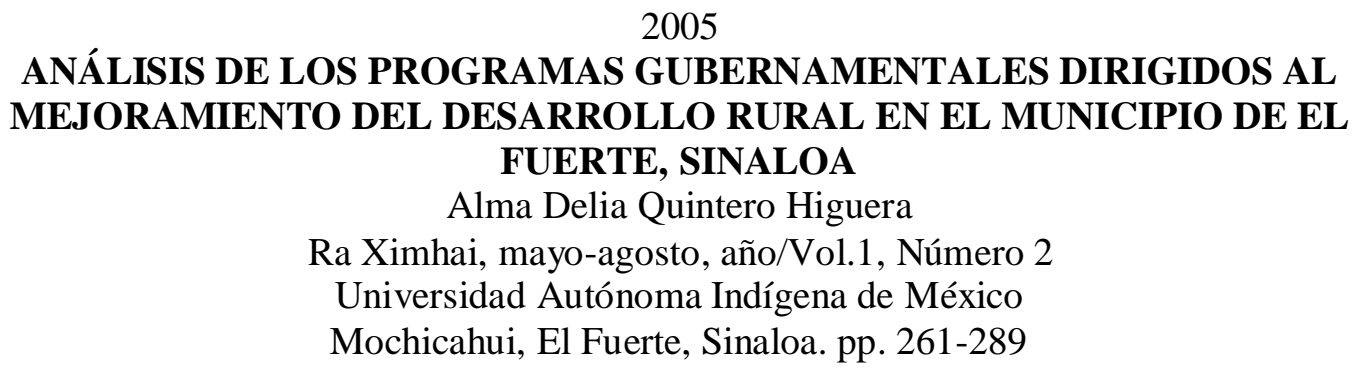

e-revist@s 


\title{
ANÁLISIS DE LOS PROGRAMAS GUBERNAMENTALES DIRIGIDOS AL MEJORAMIENTO DEL DESARROLLO RURAL EN EL MUNICIPIO DE EL FUERTE, SINALOA
}

\section{ANALYSIS OF THE GOVERNMENTAL PROGRAMS AIMED TO IMPROVE THE RURAL DEVELOPMENT AT THE MUNICIPALITY EL FUERTE}

\author{
Alma Delia Quintero-Higuera \\ Titular Académica de Grado. Licenciada en Sociología Rural. Universidad Autónoma Indígena de \\ México. Los Mochis, Sinaloa. Correo electrónico: aquintero_2@hotmail.com
}

\section{RESUMEN}

Este trabajo presenta un análisis de la operación y el impacto que han causado los programas oficiales que manejan los tres niveles de gobierno en el periodo $2002-2004$, diseñados para lograr un desarrollo humano sostenible, en la comunidad de Tehueco, El Fuerte, Sinaloa. Así mismo se realizó un análisis general de las teorías y conceptos de desarrollo y de la política social que en México se ha implementado.

Palabras clave: Desarrollo, comunidad, integración.

\section{SUMMARY}

This work presents an analysis of the operation and the impact caused by the official programs in practice at the three levels of government in the period $2002-2004$, those were designed to obtain a sustainable human development, at the community of Tehueco, El Fuerte, Sinaloa. It was also made a general analysis of the theories and concepts of development and that of the social policy that has been implemented in Mexico.

Key words: Development, community, integration. 


\section{INTRODUCCIÓN}

En nuestro país los problemas económicos, políticos y sociales, se agudizan con el paso de los años debido a la cada vez más compleja desigualdad de los grupos sociales, en la que es una lucha constante por el bienestar como persona y como grupo. México es una de las sociedades más desiguales del mundo, su nivel de desarrollo es relativamente bajo, por lo que la pobreza y la desigualdad representan un grave problema para el futuro del país.

Los gobiernos Federal, Estatal y Municipal en sus políticas de desarrollo social y humano ofrecen programas de apoyo a comunidades rurales, con el fin de colaborar en el mejoramiento del nivel de vida de los mexicanos, incrementando los niveles de educación, de salud, de vivienda, de empleo, entre otros, y con ello el bienestar y la igualdad de oportunidades para una realización plena de cada individuo, éstos, también se plantean como una respuesta a las demandas y rezagos de la población.

López (2004) dice que la realidad actual en que viven las comunidades rurales de México, exige una profunda reflexión sobre el nivel desarrollo que se ha alcanzado en el medio rural. El Estado, a través de las diferentes dependencias, plantea y diseña programas de desarrollo para el medio rural, pero es necesario también evaluar el impacto que estos programas realmente tienen en las comunidades.

\section{Concepto del desarrollo}

Este concepto es un término que en estas últimas décadas ha sido bastante utilizado, no solo en los medios políticos y económicos de casi todos los países industrializados, sino también en América Latina o países denominados pobres, subdesarrollados o tercer mundistas, para los cuales este concepto se ha convertido en un término de manejo común.

Según la enciclopedia Océano, desde el punto de vista económico se define al desarrollo como la "fase de la evolución de un país, caracterizada por el aumento de la renta nacional por habitante". Por su parte, la Comisión Asesora Ambiental de la Presidencia 
de la República del Ecuador, al referirse al desarrollo expresa que el "desarrollo es el proceso hacia el bienestar" (Atienza, 2000).

Con estas anotaciones, el desarrollo se comprende como un crecimiento hacia el mejoramiento del nivel de vida, o bienestar de los seres humanos. A partir de estas características surge la clasificación muy conocida por la cual existen países desarrollados y otros que serían subdesarrollados o tercer mundistas, es decir, aquellos que no cumplen con las características del desarrollo, entre los cuales se encuentra, obviamente, México (Aguirre et al., 2000).

\section{Desarrollo sustentable}

El término sustentable y sostenible es un término desconocido por el léxico común español, sin embargo, aparece enunciado por primera vez en el año de 1987 en el Informe de la Comisión Mundial para el Medio Ambiente, también denominado Informe Brundtland, por haber sido dirigido por la ex Ministra Noruega Gro Hariem Brundtland. De este informe surge la siguiente definición que sirve de base para anteriores definiciones: "Desarrollo sustentable es aquel que atiende a las necesidades de las generaciones presentes sin comprometer las necesidades de las futuras generaciones" (ONU, 1987).

Lo que implica que el bienestar actual de la humanidad necesariamente obliga a tomar decisiones apropiadas para no comprometer el bienestar de los que aún no nacen, es decir, que el desarrollo sustentable es un desarrollo a largo plazo, es pensar hoy y en el mañana.

A estas definiciones (Negret, 1995, citado por ONU, 2000) hace un aporte en el sentido de que el desarrollo no debe ser sostenido, sino también sostenible; considera que el crecimiento sostenido significa continuar acumulando, creciendo, proyectándose, pero como ha venido sucediendo, con dirección al caos, tal como es la proyección del crecimiento económico, que más bien se preocupa de lo cuantitativo y no de lo cualitativo. De manera que la sustentabilidad del desarrollo también es dinámica y por ende se requiere de la inteligencia humana para proyectarse en el tiempo y en el espacio. 


\section{Desarrollo humano y sostenible}

El desarrollo humano, que procede del Programa de las Naciones Unidas para el Desarrollo, y está concebido como aquél que pone al hombre en el centro del mismo, como protagonista principal del proceso de desarrollo y hacia quien tienen que ir encaminados los esfuerzos. Este enfoque hace que se tengan en cuenta las particularidades geográficas, de cada país, regionales y locales, apostando por un modelo de desarrollo propio para cada situación. Además, toma importancia el concepto de género, por la gran desigualdad en la situación de partida entre hombres y mujeres.

La definición del mismo se puede encontrar en los distintos anuales del PNUD. El Informe sobre el Desarrollo Humano 2003 sostiene que el desarrollo humano es "un proceso que permite ampliar las opciones al alcance de los ciudadanos", y en la presentación de los indicadores del desarrollo humano que propone el PNUD figura: "vigilar por el desarrollo humano: alimentar las opciones de la gente para vivir una vida larga y saludable para adquirir conocimientos, tener acceso a los recursos necesarios para un nivel de vida decente, y preservarlos a la vez para las generaciones futuras, y libre de inseguridad y equitativamente para todas las mujeres y todos los hombres" (ONU, 2000).

En definitiva, los objetivos del desarrollo humano y sostenible se pueden esquematizar en los siguientes puntos (Atienza, 2000):

1. Satisfacer las necesidades humanas básicas.

2. Lograr un crecimiento económico constante.

3. Mejorar la distribución social.

4. Atender a los aspectos demográficos.

5. Seleccionar opciones tecnológicas apropiadas.

6. Aprovechar, conservar y restaurar los recursos naturales.

7. Fomentar la igualdad entre hombres y mujeres.

8. Apostar por la educación universal. 


\section{El concepto del desarrollo sustentable y los pueblos indígenas}

Durante la historia de la humanidad los pueblos indígenas se han constituido en los protectores y guardianes de los recursos de la biodiversidad, especialmente porque desde sus orígenes han desarrollado técnicas y mecanismos de coexistencia armónica con su entorno natural. Sin embargo, en estos últimos años se vislumbra cada vez más la necesidad del respeto a la relación: desarrollo humano - sostenibilidad ambiental, como la única forma de asegurar el porvenir del planeta, sobre todo luego del deterioro, contaminación y daño que ha causado el proceso de desarrollo intensivo aplicado hasta ahora, de allí surge la necesidad de mirar el concepto de desarrollo desde la concepción indígena para proponer alternativas a futuro.

\section{Visiones sobre el desarrollo sustentable}

Dentro del presente trabajo es importante hacer un análisis respecto a cómo es visto el desarrollo sustentable desde dos visiones distintas, esto es: desde lo no indígena y desde lo indígena.

\section{a) Visión no indígena del desarrollo sustentable}

El desarrollo sustentable visto desde los no indígenas hace un énfasis principalmente en el crecimiento económico y no se orienta hacia un desarrollo humano, cultural y ético de manera más integral. Sin embargo, de alguna manera, los culturalistas y los ecosocialistas, giran, por decirlo así, el concepto sobre la base de la cultura y la naturaleza respectivamente, a fin de lograr un avance en relación a la propuesta original y superar los aspectos negativos y las críticas que el término de desarrollo sustentable generó en sus inicios, sin embrago, es evidente que de no existir conciencia, conocimiento y decisión en los actores sociales del desarrollo, es difícil que estas ideas de los culturalistas y ecosocialistas se hagan efectivas (Entrena, 1998). 


\section{b) Visión indígena del desarrollo sustentable}

Por su parte, la visión de los indígenas respecto al desarrollo sustentable, esta impregnada por la cosmovisión indígena que considera a la naturaleza como un todo, que abarca lo material, lo espiritual y humano; es la vida misma y no puede ser utilizada para enriquecerse individualmente. Esta cosmovisión tiene una serie de principios que parten de la idea de que se debe cuidar y respetar al conjunto de seres vivientes que coexisten en el ecosistema, conservar y fomentar la tierra, proteger los productos de consumo humano, para mejorar el nivel de vida de la familia y de la comunidad; proteger los recursos no renovables, incentivar a la comunidad para que cuide su propio ambiente, socializar a nivel de la organización y las comunidades acerca de la conservación del entorno como garantía de una vida digna tanto para las actuales generaciones como para las futuras (Caron, 1996).

\section{Desarrollo rural}

Las concepciones de desarrollo rural se han ido modificando en la medida en que se percibe con mayor claridad la complejidad y diversidad de la realidad y se evidencian las restricciones y posibilidades de sus explicaciones y alcances.

El desarrollo rural se entiende hoy como un proceso de mejoramiento de las condiciones de bienestar de la población rural, reconociendo la contribución que el medio rural hace al bienestar general de la población en su conjunto (ya sea urbana o rural). El desarrollo rural se asume como un proceso histórico de transformación, en el cual se considera la pluralidad de actores en una diversidad de condiciones y posiciones. Ello significa que se reconoce que el Estado y las instituciones participan en el proceso, pero los actores fundamentales son los pobladores, que además no son una población homogénea.

En la nueva conceptualización del desarrollo rural está presente el reconocimiento de la diversidad y heterogeneidad en sus diferentes manifestaciones, lo cual exige políticas, planes, programas y proyectos diferenciados, que respondan a una visión con equidad de género y que puedan dar respuesta a una realidad heterogénea, especialmente en el sector rural. 


\section{Los paradigmas actuales del desarrollo rural en México}

El paradigma dominante en materia de desarrollo rural sigue privilegiando la idea de modernizar el medio rural como estrategia de progreso en todos sentidos: productivos, educativos, tecnológicos, etc.

Una prioridad importante para los habitantes del mundo rural es el mantenimiento de su estilo de vida. Ello significa que no desean un desarrollo que implique transformaciones profundas en las áreas rurales, sino políticas de desarrollo rural que les permitan permanecer en el espacio rural con unos servicios y equipamientos más próximos a los que disfrutan las zonas urbanas. La naturaleza de las futuras políticas de desarrollo debería depender de una reflexión previa necesaria sobre cuáles deben ser los diferentes modelos de espacios rurales de futuro y quién puede y debe tomar estas decisiones, y el papel que los agentes rurales tienen en estas decisiones (López, 2004).

La participación del Estado puede complementar las estrategias como una alternativa eficiente en las políticas públicas, en la idea de un desarrollo rural, además de que en la instrumentación de políticas públicas por medio de los programas se contemplen las formas en que las personas oriundas de los lugares viven su cotidianidad, y además de tomar en cuenta también las acciones que llevan a esos sujetos a solucionar problemas y seguir preservándose como sociedad, pese a las constantes adversidades (Herrera, 2004).

\section{Política social y la nueva política social}

Históricamente la política social de México está basada en el crecimiento económico del país por lo que el bienestar de la población queda subordinado al desempeño económico y reiteradamente a un futuro incierto.

Vargas (2004) comenta que la política social siempre estaba involucrada con la solución de problemas del sistema social más que del económico, tales como los derechos sociales de los ciudadanos, las cuestiones de la administración social, las formas y la racionalidad de la redistribución de la riqueza y el servicio social. 
De acuerdo con este autor la nueva política social no es nada mas que la privatización de bienes y satisfactores sociales que atrasan aun más a los menos afortunados de manera que no les permite tener parte alguna en el desarrollo y señala que el papel del Estado en la "nueva política social", en el mejor de los casos, es la regulación, inspección y provisión de las deficiencias de las actividades del sector privado.

Se requiere urgentemente trabajar en la construcción de un proyecto de sociedad compartido, que reconozca la diversidad, la heterogeneidad, la complejidad de las relaciones sociales, así como la necesidad de una convivencia que ofrezca oportunidades de desarrollo para todos los mexicanos, en un marco de respeto, tolerancia, corresponsabilidad e integración social que permita superar las desigualdades y los desequilibrios sociales y avanzar en la construcción de la necesaria ciudadanía social.

\section{Programas gubernamentales de desarrollo rural en México}

La Federación anuncia un catálogo de programas bastante notable. Entre ellos destacan: el Programa de Desarrollo Humano Oportunidades; el Programa de Abasto Social de Leche a cargo de Liconsa; el Programa Jóvenes por México; el Programa Opciones Productivas; el Programa Apoyo a la Palabra; el Programa Acompañamiento y Formación Empresarial; el Programa Agencias de Desarrollo Social; el Programa Crédito Productivo para Mujeres; el Programa Escuelas de Calidad; el Programa Integración Productiva; el Programa de Empleo Temporal; el Programa del Fondo Nacional de Fomento a las Artesanías; el Programa para el Desarrollo Local de Micro regiones; el Programa Mujeres Jefas de Familia; el Programa para el Desarrollo de los Pueblos y Comunidades Indígenas; el Programa de Conversión Social; el Programa de Cuadros Indígenas y Fortalecimiento al Capital Social y el Programa Desarrollo de las Culturas Indígenas; el Programa Procampo y el Programa Diconsa, por mencionar algunos.

En cuanto al gobierno de la entidad, denotamos el Programa para la Sostenibilidad de los Servicios de Agua Potable y Saneamiento en Comunidades Rurales en Sinaloa; el Programa de Empleo Temporal; el Programa de Opciones Productivas; el Programa 
Apoyo a la Palabra; el Programa de Desarrollo Social a Jornaleros Agrícolas; el Programa de Desarrollo Rural de la Alianza Contigo; el Programa de Apoyo a los Proyectos de Inversión Rural; el Subprograma de Desarrollo de Capacidades en el Medio Rural; y el Subprograma de Fortalecimiento a Empresas y Organizaciones Rurales.

El Ayuntamiento, por su parte interviene en la transferencia o intermediación de varios programas, como lo son:

Jóvenes por México.

Oportunidades Productivas.

Apoyo a la Palabra.

Empleo Temporal.

Estímulos a la Educación Básica, y

Vivah

Este trabajo se realizó con el objetivo de analizar cada uno de los programas gubernamentales dirigidos al desarrollo rural que se manejan en Tehueco en el Municipio de El Fuerte, Sinaloa, México y determinar los elementos fundamentales que hacen posible o no la aplicación y efectividad de estos programas, incluyendo los trámites con el gobierno Federal, Estatal, Municipal y local, considerando de antemano la participación activa de la gente que habita en el Municipio. Con el fin de dar respuesta a interrogantes como: ¿Cuáles son los programas gubernamentales destinados a contribuir en el mejoramiento de la calidad de vida en el medio rural?, ¿Por quién o quiénes son coordinados?, ¿Qué problemática se presenta en cuanto a la difusión y aplicación de los programas de gobierno?, ¿Cuál ha sido el impacto que estos programas han causado en la comunidad de Tehueco?. 


\section{MATERIALES Y MÉTODOS}

\section{Localización del área de estudio}

La comunidad de Tehueco es un asentamiento pequeño de aproximadamente 1,000 habitantes que se encuentra ubicada a 23 kilómetros de la ciudad de El Fuerte, Sinaloa, México (Figura 1). Al norte limita con el río Fuerte, al sur con el ejido Las Estacas, al oriente con el ejido Montoya y al poniente con las tierras particulares del señor Clemente Soto Peña.

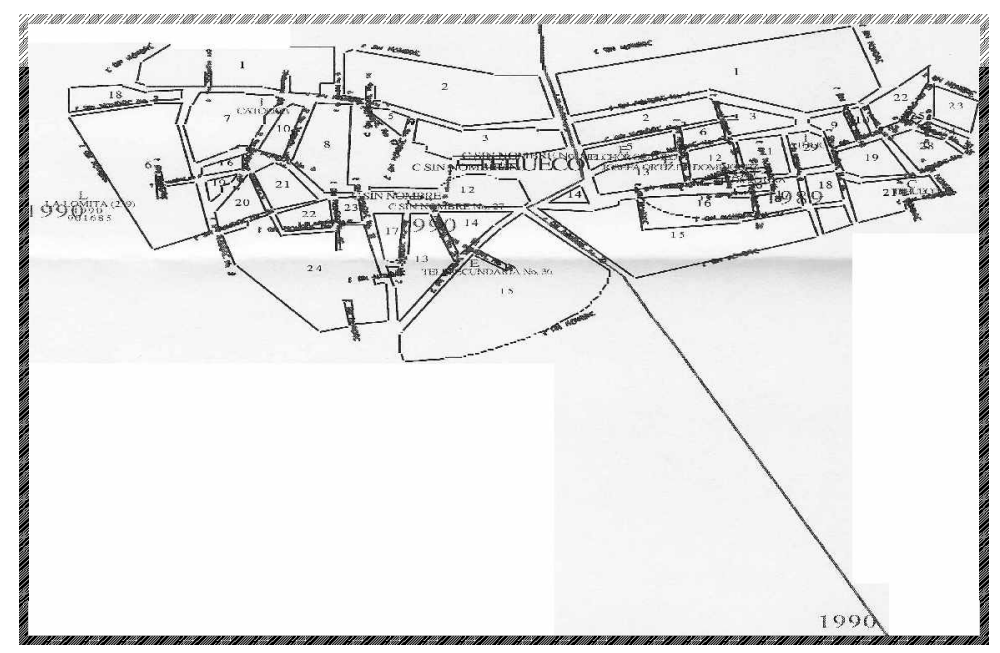

Figura 1. Plano de la comunidad de Tehueco.

Miguel Quintero (1997), en su libro “Tehueco Tradiciones y Costumbres” explica el origen de la comunidad de la siguiente manera: sin encontrar archivo bibliográfico, pero considerando las costumbres que existen y la fundación de la Villa de San Juan Bautista de Carapoa (hoy El Fuerte de Montesclaros) en 1564, se considera que el pueblo de Tehueco ya era habitado desde esa época.

Metodología

A partir de un enfoque metodológico que nos permite profundizar en los aspectos sociointerculturales de una realidad social, en este trabajo se expone el estudio de caso en la comunidad de Tehueco, Municipio de El Fuerte, Sinaloa, México. A través de la revisión y el análisis de los documentos bibliográficos se observaron la forma específica 
que asumieron los diferentes actores, tanto a nivel local como federal. Además a través de la investigación-acción y de investigación etnográfica, fundamentalmente por la vía de recopilación de relatos de los pobladores de la comunidad fue posible integrar la dimensión del desarrollo sostenible en el análisis de la comunidad.

Los relatos de vida nos permitieron un acercamiento multidimensional a los procesos de cambio en un espacio rural localizado. De esta manera, se recuperaron las experiencias locales de una población rural y que nos permitieron analizar el impacto que han tenido los programas oficiales de gobierno que se han aplicado en los últimos tres años, en la comunidad y sus logros alcanzados con estos programas.

Para conocer la opinión de la comunidad acerca de la aplicación de los programas gubernamentales que se han aplicado en Tehueco en los últimos años, se realizó un muestreo simple al azar para proporciones con un error $\pm 5 \%$ y una confiabilidad del 95\%; tomando como marco muestral el número total de habitantes.

La fórmula empleada para calcular el tamaño de la muestra fue (Gómez, 1977):

$$
\mathrm{n}=\frac{\mathrm{N}(\mathrm{Z} \alpha / 2)^{2} \mathrm{p}_{\mathrm{n}} \mathrm{q}_{\mathrm{n}}}{\mathrm{N}(\mathrm{d})^{2}+(\mathrm{Z} \alpha / 2)^{2} \mathrm{p}_{\mathrm{n}} \mathrm{q}_{\mathrm{n}}}
$$

Donde:

$\mathrm{n}=$ Es el número total de la muestra.

$\mathrm{N}=$ Número total de la población.

$Z_{\alpha / 2}=$ Confiabilidad.

$\mathrm{d}=$ Precisión .

$\mathrm{p}_{\mathrm{n}}=$ Proporción de la variable de interés.

$\mathrm{q}_{\mathrm{n}}=$ Proporción de la variable complementaria. 


\section{RESULTADOS Y DISCUSIÓN}

\section{La cultura Yolem'me mayo en la comunidad}

Tehueco se caracteriza por ser uno de los centros ceremoniales de los mayo de Sinaloa, Aproximadamente 543 personas de la población pertenecen a la etnia mayo y las 357 restantes son mestizos (yoris) (Figura 2).

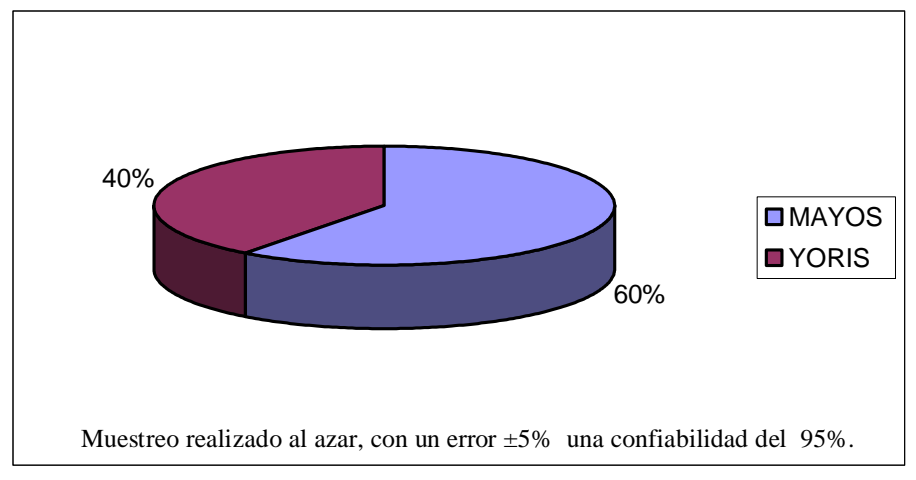

Figura 2. Porcentaje de Mayos y Yoris respecto a la población total.

La vida ceremonial de los mayos de Tehueco se expresa a lo largo de todo el año en diversas fiestas y ceremonias que este grupo práctica, donde todas estas celebraciones son un sincretismo de la doctrina de la religión católica con la religión autóctona.

Lamentablemente, la devoción por la cultura mayo está perdiendo fuerza en las nuevas generaciones de la comunidad de Tehueco. En una entrevista realizada al Sr. Miguel Alonso Quintero Armenta, escritor del libro "Tehueco Tradiciones y Costumbres", quien se ha caracterizado por su incansable lucha por preservar la cultura mayo en la región nos dice:

"la cultura de los mayos está en total decaimiento, y creo que uno de los principales factores que influye es que la política se metió a las comunidades yoremes y desde entonces se hecho a perder todo, por que los lideres ya no quieren ser lideres indígenas sino lideres políticos, la migración de los jóvenes de la comunidad a otras ciudades y a 
EE.UU., además de que otras religiones han penetrado en la comunidad y también son factores determinantes en el decaimiento de la cultura mayo..."

Preguntamos a los habitantes de la comunidad qué piensan de la cultura mayo en su comunidad y la respuesta que dio toda la población fue que se está perdiendo y que si no se hace algo pronto va a desaparecer, pues sólo hay en la comunidad aproximadamente 20 personas que conocen bien la cultura, todos ellos avanzados de edad, sobre lo cual podemos suponer que dentro de 10 a 20 años más no habrá nadie que se interese por practicarla y difundirla. La Figura 3 nos muestra, enumeradas de mayor a menor orden de importancia, las principales causas señaladas por la población del porqué se esta perdiendo la identidad indígena.

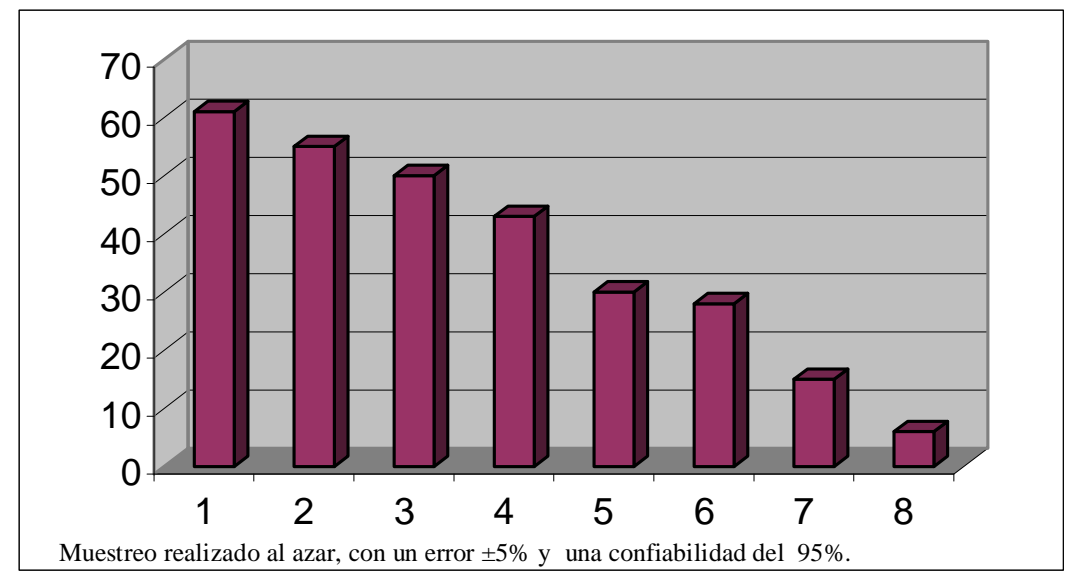

1.- Desinterés de los padres, 2.- Influencia de medios de comunicación, 3.- Vergüenza, 4.- Migración, 5.- Invasión de personas ajenas a la cultura, 6.- Falta de utilidad, 7.- Discriminación, 8.- Otra.

Figura 3. Principales causas de la pérdida de identidad.

Tehueco es una de las 7 sindicaturas que conforman el municipio de El Fuerte (Figura 4), también cuenta con un comité de desarrollo integrado por hombres y mujeres con la responsabilidad de luchar por el desarrollo de la misma, gestionando apoyos ante las instancias correspondientes y organizando a la población para su mejor aprovechamiento. 


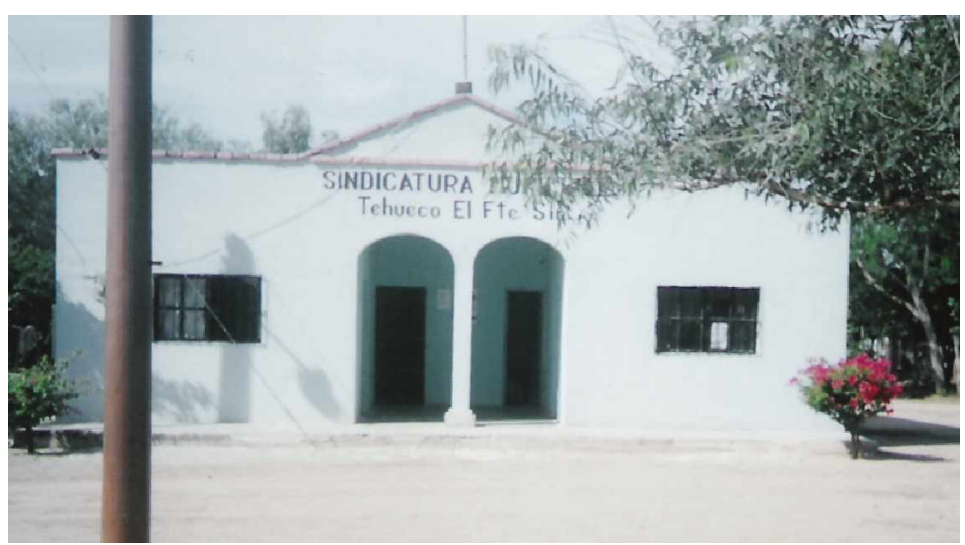

Figura 4. Sindicatura de Tehueco.

El ejido de Tehueco ha tenido un total de 147 ejidatarios y una distribución parcelaria de 3, 630 ha de las cuales sólo 1,500 ha son aptas para el cultivo y el resto son de agostadero.

Debido a la precaria situación económica que se vive en la comunidad, la mayoría de los ejidatarios se han visto en la necesidad de rentar o vender sus tierras. De acuerdo a los datos obtenidos en el ejido Tehueco, 30 de esos 147 ejidatarios que lo conformaban han optado por vender su derecho total de tierras. Por lo cual, actualmente sólo cuenta con 117 ejidatarios, de los cuales 77 de ellos han vendido una parte de su derecho y 30 de ellos aún lo conservan completo, tal como lo muestra la Figura 5.

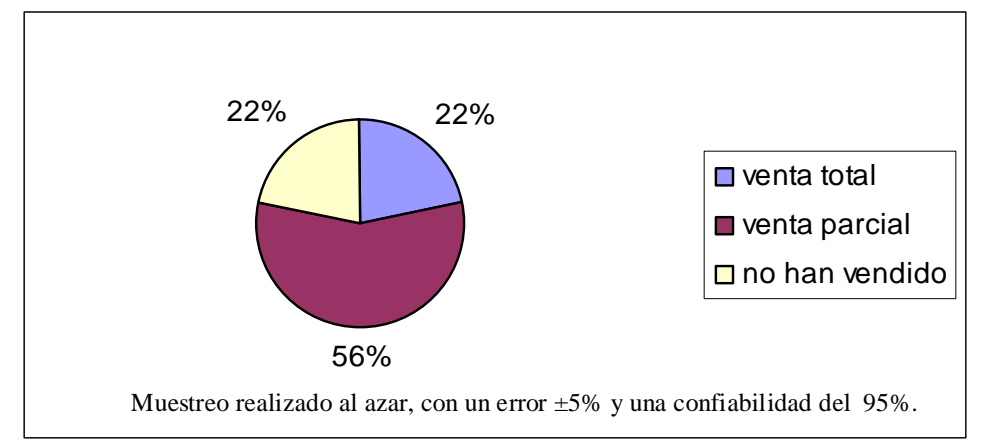

Figura 5. Venta de la parcela.

De estos 117 ejidatarios que actualmente conforman el ejido el Tehueco; 100 de ellos se ven en la necesidad de rentar sus parcelas a otros agricultores debido a los altos costos que implica una producción y 17 de ellos las siembran con diversos cultivos, ya sean para comercialización o para autoconsumo. 


\section{Situación socioeconómica de la Comunidad de Tehueco}

La principal actividad que se realiza en la comunidad para sostenimiento de la familia es el trabajo en el campo, como jornaleros, ya que según los datos un $62 \%$ de la población se dedica a esta actividad (Figura 6), un 19\% se emplean como choferes (en una granja avícola) o cargadores, etc., un $11 \%$ de la población obtiene el ingreso de la familia de otra forma, es decir, algunos de ellos emigran a otras ciudades o Estados Unidos. Únicamente el $8 \%$ restante tiene su propio negocio, dentro de esta categoría se encuentran los comerciantes y productores de la comunidad.

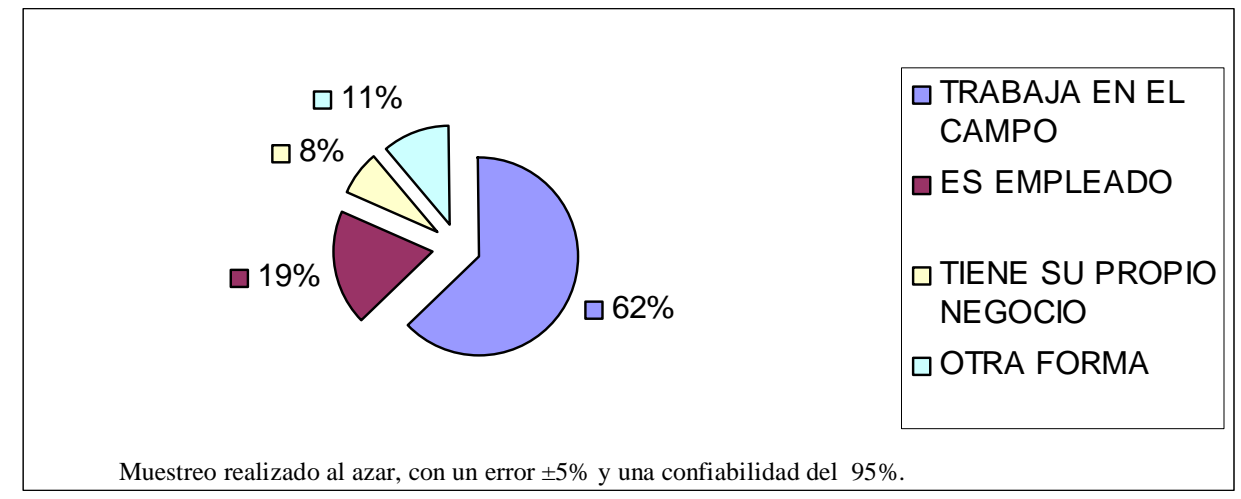

Figura 6. Actividad Económica.

Todo esto nos indica que la situación económica de la comunidad es crítica, como bien sabemos, el trabajo en el campo es pagado con un sueldo mínimo que no alcanza a una familia ni para suplir las necesidades básicas de la misma, como son la alimentación, la salud y la educación.

Como hemos visto anteriormente, la principal actividad económica de la comunidad es el trabajo asalariado en el campo; que se refleja en el promedio de ingreso semanal de la familia, que es el salario mínimo (Figura 7). 


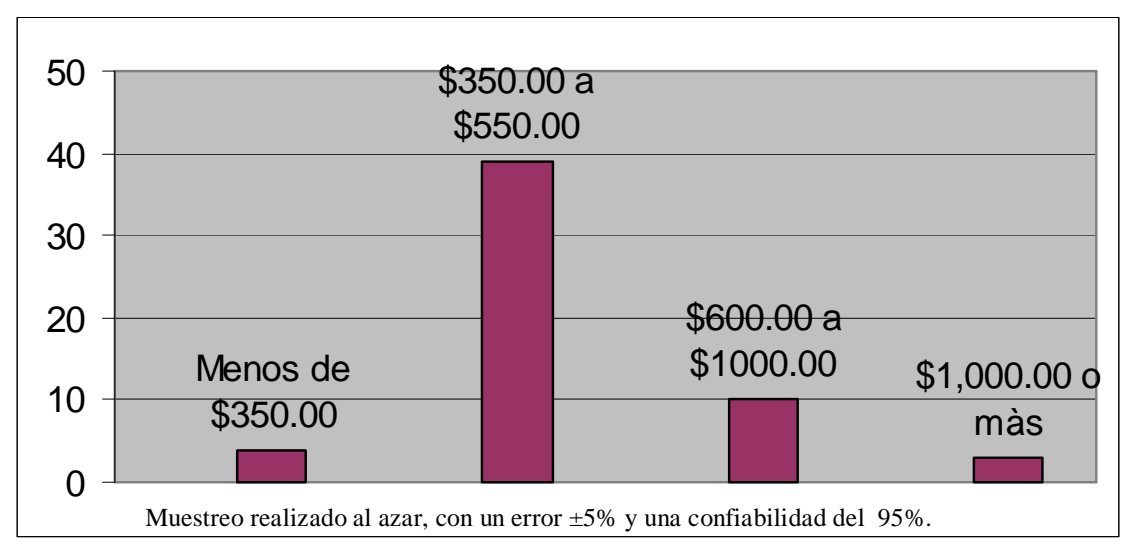

Figura 7. Ingreso Semanal a la Familia.

Tomando en cuenta que el promedio de habitantes por vivienda en la comunidad varia entre 4 y 10 personas en cada casa y que el número promedio de trabajadores por vivienda es de 2 a 4, podemos comprobar que es crítica la situación económica que están viviendo las familias de Tehueco; ya que un salario mínimo no es suficiente para suplir las necesidades primarias de una familia (Figura 8).

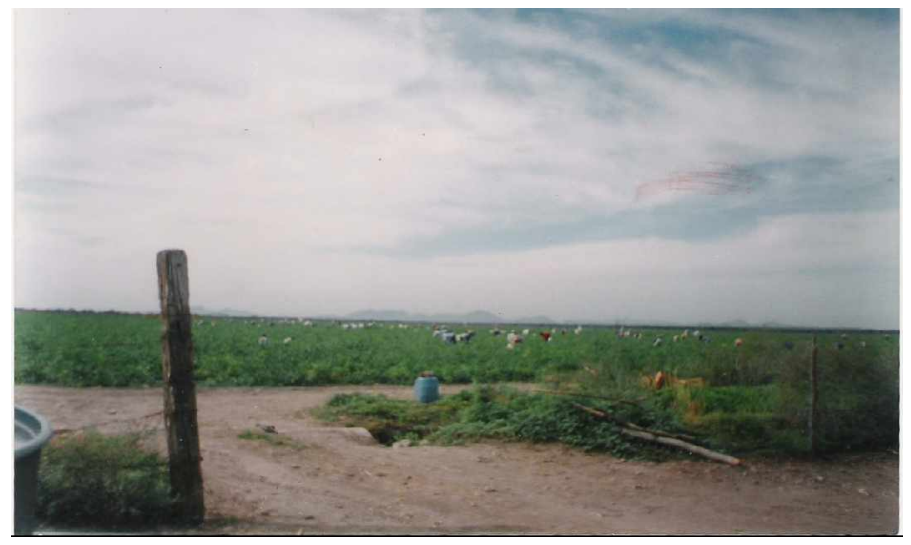

Figura 8. Jornaleros de Tehueco.

El señor Javier Quintero ex Síndico de la comunidad y quien se ha dedicado la mayor parte de su vida a la agricultura nos dice que: desde que yo tengo uso de razón la principal fuente de ingresos a la familia era la agricultura y yo me había dedicado a esto siempre, pero en estos últimos años no costea sembrar las parcelas porque los costos de la producción son muy caros la maquinaria, la semilla, los químicos y todo esta muy caro, solo la gente de dinero son los que pueden costear todos estos gastos, por que con la situación del TLC los productores mexicanos siempre salimos 
perdiendo, es por eso que mi familia y yo nos iremos a buscar otras oportunidades de mejorar nuestra situación económica en Estados Unidos.

La ganadería es una actividad que practican muy pocas personas de la comunidad sólo 25 personas se dedican a la crianza y producción de ganado vacuno y la cantidad de ganado que poseen va desde 10 hasta 200 reses aproximadamente.

La pesca no es muy común en la comunidad a pesar de que la misma está ubicada a la orilla del Río Fuerte, algunas personas que la realizan lo hacen muy eventualmente y solo para autoconsumo, es por ello que no es una actividad importante en la economía de Tehueco.

Una de las principales consecuencias de la situación económica que enfrenta esta comunidad se ve reflejada en factores tan importantes e indispensables para el desarrollo de la misma; como la educación, que en la población entrevistada es muy común el bajo nivel de formación académica que han alcanzado estas personas, como se muestra en la Figura 9.

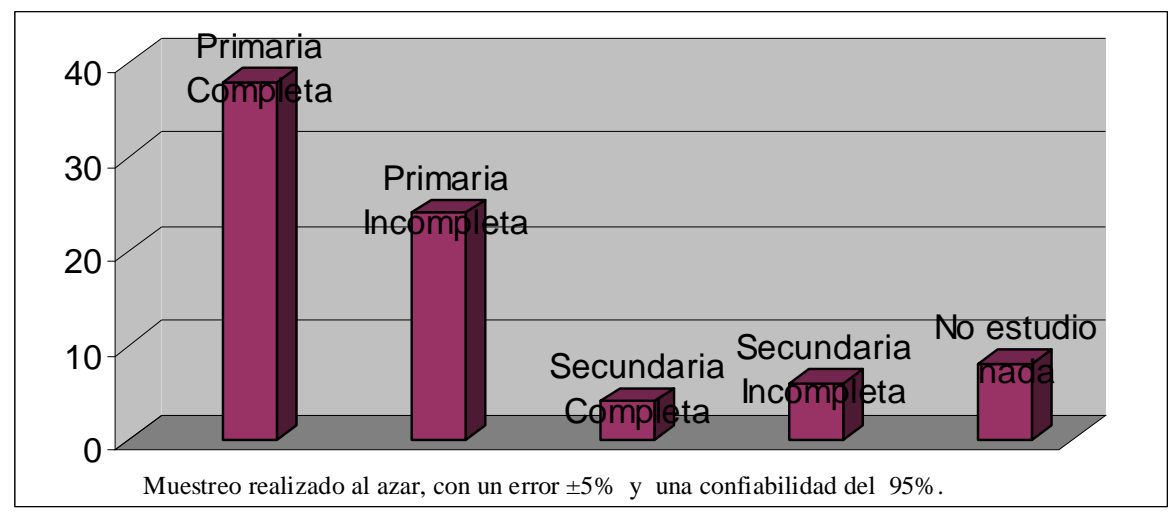

Figura 9. Nivel de Estudios.

La principal causa señalada de esta situación, es la falta de recursos económicos para seguir estudiando, pues la mayoría de la población dice que en aquellos tiempos sus padres no tenían dinero para mandarlos a la escuela y comprarles sus cuadernos y demás útiles y los padres los sacaban de la escuela para que les ayudaran a trabajar para el sostenimiento de su familia. Algunas personas de la comunidad consideran que los apoyos a la educación que están recibiendo algunos niños y jóvenes de la comunidad es 
un gran paso en la misma. Hoy muchos adultos han terminado su educación primaria y secundaria gracias al Instituto Nacional para la Educación de los Adultos (INEA), que han impartido clases a muchos de los adultos que habitan en Tehueco.

Es importante recalcar que en los últimos años se les ha brindado a los jóvenes en edad de estudiar, oportunidades de continuar los estudios por medio de becas e incentivos, para que ellos puedan hacer una carrera profesional y con ello contribuir al desarrollo de su comunidad. Actualmente la comunidad cuenta con los tres niveles de educación básica que es preescolar, primaria y telesecundaria y en los últimos 10 años, aproximadamente un $40 \%$ de los jóvenes que terminan estos niveles de educación básica continúan estudiando la preparatoria o algún curso técnico y un $20 \%$ de ellos estudia una carrera profesional.

\section{Análisis de los programas gubernamentales en Tehueco}

Según la encuesta realizada podemos notar con claridad (Figura 10) que un $86 \%$ que representa la mayoría de la población visitada, conoce o ha escuchado hablar de los programas gubernamentales que se han aplicado en la comunidad de Tehueco. El 14\% restante dice que no los conoce, que tal vez haya escuchado hablar de ellos, pero ignora en qué consisten. Este tipo de información básica que es muy importante que toda la población de la vcomunidad esté enterada y esté dispuesta a integrarse en su aplicación.

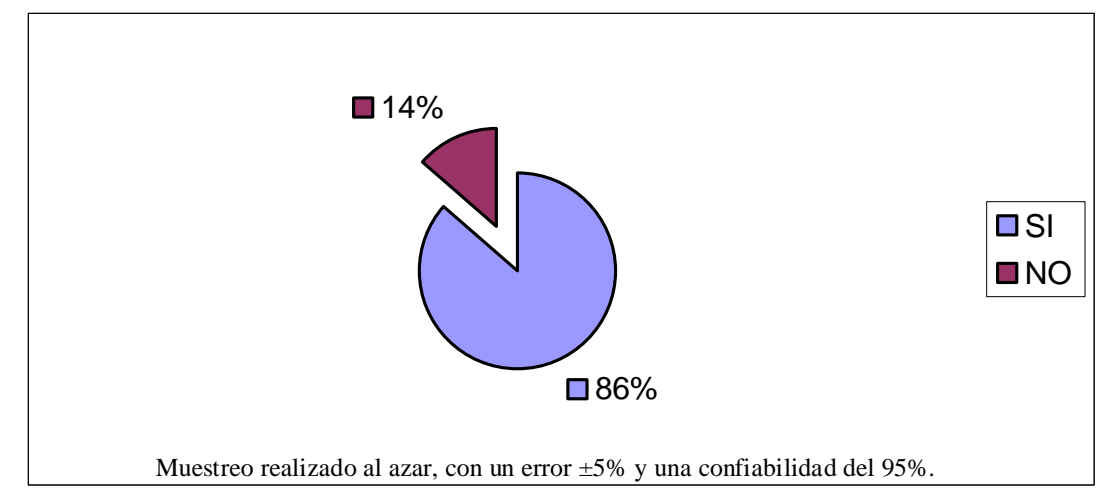

Figura 10. Conocimiento de programas gubernamentales.

Al señalar cuáles son los programas que conoce la población las respuestas fueron ordenadas de mayor a menor orden de importancia (Figura 11). 


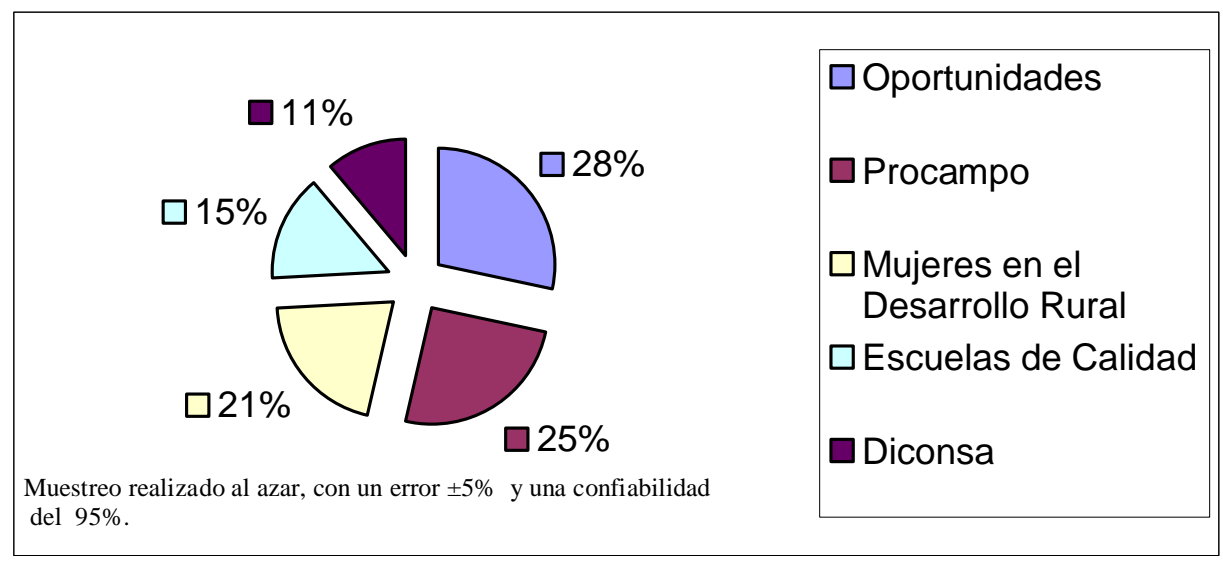

Figura 11. Programas conocidos.

Es interesante notar que de la gran oferta de programas de gobierno encaminados al desarrollo rural, el registro de acciones en la comunidad de Tehueco, únicamente ha identificado cinco: 1.- el Programa de Desarrollo Humanos Oportunidades; 2.- el Programa Escuelas de Calidad; 3.- el Programa de Apoyo a los Proyectos de Inversión Rural; 4.- el Programa Procampo y 5.- el Programa Diconsa. No mencionamos aquí los programas de educación bilingüe ni seguro popular que no asociamos con políticas de desarrollo comunitario.

1) El Programa de Desarrollo Humano Oportunidades, es un programa coordinado por la Secretaría de Desarrollo Social (SEDESOL), Secretaría de Educación Publica (SEP) y la Secretaría de Salud (SSA), que articula incentivos para la educación, para la salud y para la nutrición, el Programa tiene como objetivo apoyar a las familias que viven en condición de pobreza extrema con el fin de potenciar las capacidades de sus miembros y ampliar sus alternativas para alcanzar mejores niveles de bienestar, a través del mejoramiento de opciones en educación, salud y alimentación.

Éste programa esta aplicándose en la comunidad de Tehueco desde 1998, actualmente son beneficiadas un total de 102 familias de la comunidad. El esquema de operación de este programa consta de tres componentes esenciales que son la educación, salud y alimentación. Las familias de la comunidad reciben una ayuda económica para alimentación y otra cantidad para educación que las madres de familia emplean para comprar uniformes y útiles escolares a sus hijos, y en cuanto a la salud, los niños acuden cada dos meses a consulta con el medico familiar quien vigila que estos niños 
estén sanos y bien nutridos; los adultos de la familia acuden a consulta cada seis meses y las mujeres embarazadas una vez al mes, para vigilar el estado de salud del niño y de la madre durante todo el embarazo y lactancia.

Entrevistamos a algunas familias beneficiarias del programa de donde anotamos lo siguiente:

La Sra. Concepción Peña Gil nos dice “el programa Oportunidades ha beneficiado bastante a mi familia porque muchas veces no nos alcanza el dinero para vestir a nuestros hijos o para llevarlos con el doctor o para comprarles sus cuadernos y desde que tengo esta ayuda, aunque no es mucha, cuando nos llega el dinero puedo comprarles a mis hijos su ropa, sus útiles y lo que a ellos les gusta comer. Además cada 2 meses los revisa el doctor y yo me quedo tranquila de saber que mis hijos están bien de salud y bien nutridos"

Como ésta señora son muchas las madres de familia que se declaran satisfechas con la implementación de este programa. Éste apoyo se otorga a cada familia en un periodo de tres años, luego se les retira la ayuda para dar oportunidad a otras familias que no lo han recibido y que necesitan de él. Sin embargo, podemos notar que hay un problema en el método de selección de familias beneficiadas, ya que muchas de las familias que realmente lo necesitan no tienen este apoyo y otras que tienen la manera de satisfacer estas necesidades por su propia cuenta si la tienen.

A pesar de esto, el impacto que este programa ha causado en la comunidad ha sido muy alto; pues se puede ver claramente el grado de satisfacción que existe en estas familias. Vemos que han respondido con alto grado de responsabilidad al cumplir con todas las obligaciones que al ser beneficiarios de este programa implica, como la de mantener limpia su casa, su comunidad, el buen aprovechamiento de los recursos otorgados, y la puntual asistencia a las citas médicas de la familia.

2) El Programa Escuelas de Calidad, PEC, forma parte de la política nacional de reforma de la gestión educativa, que busca superar diversos obstáculos como son el estrecho margen de la escuela para tomar decisiones, el desarrollo insuficiente de una 
cultura de planeación y evaluación en la escuela, los excesivos requerimientos administrativos que limitan a los directivos escolares para ejercer un liderazgo efectivo, la escasa comunicación entre los actores escolares, el ausentismo, el uso poco eficaz de los recursos disponibles en la escuela, la baja participación social y las deficiencias en infraestructura y equipamiento.

Éste programa está operando en la comunidad desde el año 2002 en la escuela Telesecundaria \#36 (Figura 12). Con la aplicación de este programa en la telesecundaria se ha logrado darles un uso eficaz a los recursos con que cuenta la misma para mejorar las condiciones físicas (en cuanto a la construcción de nuevas aulas y pintura), así como su sistema educativo pues han logrado establecer un centro de computo donde se cuenta con un total de 5 equipos, que les han permitido mejorar la calidad de educación que ahí se imparte.

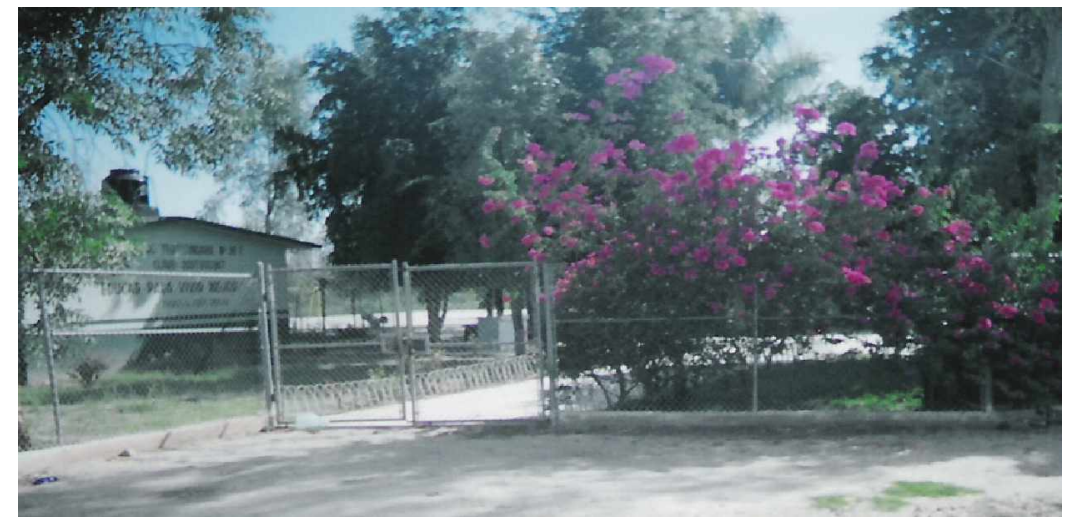

Figura 12. Telesecundaria \#36 de la comunidad de Tehueco.

Sobre esto preguntamos a la Sra. Isabel Quintero encargada del comité de vigilancia de este programa quien nos dice: éste programa ha sido de gran beneficio para los estudiantes de telesecundaria de la comunidad; pues les permite tener una educación de mejor calidad a la que tenían antes y así fundar las bases que les ayudarán a ser profesionales exitosos en un futuro, además que nos ayuda a aprovechar de la mejor manera los recursos con los que cuenta la escuela para el mejoramiento de la misma, ya sea físicamente o materiales educativos como libros, computadoras y otras cosas”.

De acuerdo con lo expresado, preguntamos a algunos padres de familia acerca de su opinión sobre esto, los cuales coincidieron en que ha sido un beneficio muy grande 
para sus hijos, sus familias y su comunidad. Esto motiva a sus hijos a continuar sus estudios: lo malo, menciona uno de ellos, es que somos gente que vive del diario que ganamos en el campo y nos preocupa el no poder seguir sosteniendo el estudio de nuestros hijos, por que esa ayuda a la escuela si es buena pero sí el gobierno no se preocupa por generar empleos en la comunidad va a ser la misma, por que nuestros hijos no van a poder seguir estudiando y como todos los demás se van a dedicar toda su vida al campo o a la casa.

3) El Programa de Apoyo a los Proyectos de Inversión Rural, en 2002 llamado Mujeres en el Desarrollo Rural, es un subprograma que se manejó en el año 2002 mediante el Programa de Apoyo a los Proyectos de Inversión (PAPIR) coordinado por la Secretaría de Agricultura, Ganadería, Desarrollo Rural, Pesca y Alimentación (SAGARPA), que consiste en fomentar la inversión en bienes de capital de la población rural elegible a través del apoyo para la puesta en marcha de proyectos productivos que posibiliten la aplicación de tecnologías apropiadas, la reconversión productiva, el acopio, acondicionamiento y transformación; para fortalecer la comercialización de la producción primaria, la generación de empleo rural y de servicios, así como su posicionamiento en los mercados.

Por medio de este programa se benefició a un grupo de 6 mujeres habitantes de la comunidad de Tehueco en la instalación y fortalecimiento de una microempresa rural denominada "Mercería la Ilusión" (Figura 13), las cuales trabajan de manera muy responsable en el mantenimiento de la mercería aprovechando el apoyo que han recibido del gobierno mediante este programa.

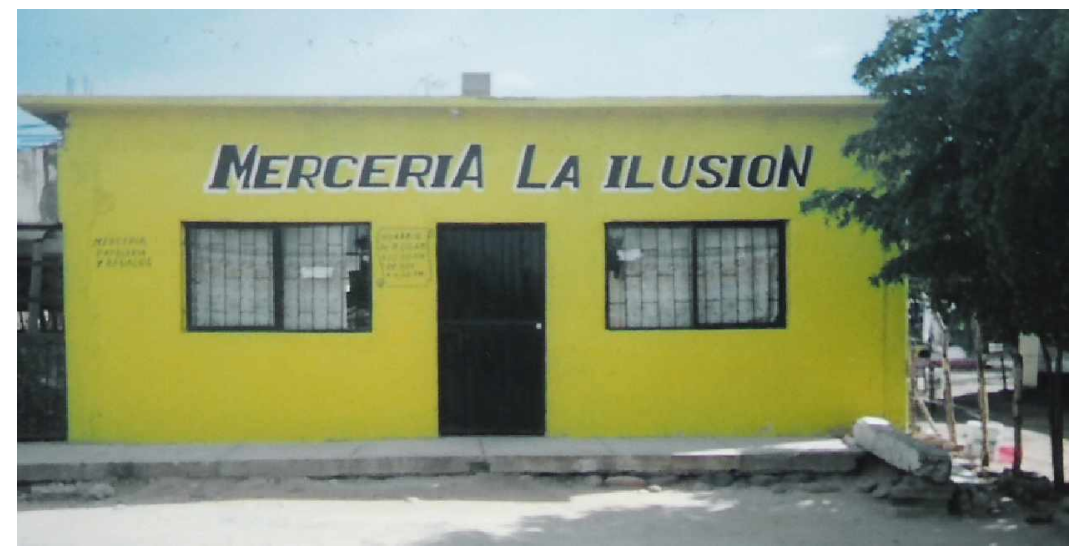

Figura 13. Mercería de la comunidad de Tehueco. 
Para conocer el impacto que este programa ha causado en la comunidad entrevistamos a algunas socias de la mercería y a personas habitantes de la comunidad.

La señora Patricia Aída Gaxiola Leyva quien es la representante legal de este grupo de mujeres que son socias en este proyecto, nos dice lo siguiente: nosotros no creíamos que el gobierno nos fuera a dar un apoyo de este tipo, cuando vinieron a proponernos que participáramos en este proyecto, si aceptamos, pero dudamos que fuera cierto por que sabemos que es muy difícil que el gobierno mande apoyos de este tipo, ahora que lo vemos hecho realidad estamos muy contentas por este apoyo y le estamos trabajando duro para no perder nuestro negocio, ya que nos ha beneficiado muchísimo tanto a nuestras familias como a la comunidad.

La señora Justina Higuera habitante de la comunidad de Tehueco nos dice: yo creo que la mercería ha sido de mucho bien para el pueblo en la economía familiar, por que me acuerdo que antes si necesitábamos algún hilo o cierre o aguja, teníamos que ir hasta El Fuerte a comprarlos y era doble gasto por el pasaje que teníamos que pagar. Ahora nos ahorramos tiempo y dinero por que aquí tenemos lo que necesitamos muy a la mano, además algunas cosas están más baratas aquí que en El Fuerte, por eso yo creo que nos ha beneficiado mucho y más a quienes somos costureras o a quienes tenemos niños en la escuela”.

De acuerdo a lo anterior se observa que, efectivamente, la implementación de este programa ha beneficiado a la comunidad generando empleo a seis mujeres. Los productos que ofrecen son de primera necesidad y el servicio que dan es de calidad.

También consideró - la Sra. Huiguera- importante señalar: que hace apenas unas semanas llegó a la comunidad un proyecto productivo más, por medio del mismo programa (PAPIR), que consistía en una zapatería. Los encargados de la aplicación del programa llegaron a la comunidad y sólo dieron unas horas para que se formara el grupo que deseaba participar en el proyecto, por lo que no hubo resultados positivos ya que no dieron tiempo de reunir a toda la comunidad e informarles a cerca de esto y nadie de las personas que se enteraron quiso hacerse responsable, se perdió ese proyecto y con él la oportunidad de lograr un avance más por medio de la generación de empleos en la comunidad. 
El Programa Procampo se ha implementado en la comunidad por muchos años, consiste en otorgar a los productores del ejido cierta cantidad de dinero por hectárea como una ayuda para contribuir en los gastos que implican los cultivos. Desafortunadamente este apoyo es insuficiente para suplir los gastos que producción de un cultivo implica, por lo que la mayoría de los ejidatarios se ve en la necesidad de rentar sus tierras a otros productores para que las trabajen y son ellos los que reciben este beneficio de procampo. Por otro lado, de acuerdo a investigaciones realizadas en la comunidad vemos que algunos ejidatarios que no rentan sus tierras reciben este apoyo y no lo utilizan en sus cultivos o para bien de la familia, sino que optan por satisfacer sus vicios de alcoholismo o los malgastan en gastos superfluos.

Por lo anteriormente expuesto es observable que existe una deficiencia en la aplicación de este programa, en el aspecto de vigilancia y en el empleo de recursos otorgados al productor, ya que la mayoría de las veces no se emplea adecuadamente para lo que es enviado ni tampoco acarrea ningún beneficio a las familias ni a la comunidad.

El Programa Diconsa opera en la comunidad desde el año 1980. En Tehueco se encuentra ubicado el almacén de alimentos que se distribuye a las tiendas Diconsa, y a los albergues indígenas que hospedan niños de bajos recursos de educación primaria y secundaria del norte del Estado. Es curioso notar, como el programa que ha estado funcionando en la comunidad por más tiempo, resulte ser el menos conocido por la población. Por este motivo nos llevó a ahondar la investigación. La acción básica de este programa en la comunidad ha sido la generación de empleos para los habitantes de la misma y permitirles una comercialización segura de las cosechas que en el ejido se producían, propósito que se cumplió en los primeros años de su funcionamiento y de un tiempo a la fecha los empleados de la comunidad han sido reemplazados por personas totalmente ajenas a la misma y la comercialización de sus productos fue siendo cada vez más difícil, es por eso que en los últimos años, la comunidad ha perdido contacto con el funcionamiento de este programa.

Por otra parte, consideradas estas acciones como manera y política gubernamental para atender las demandas sociales de la comunidad, es observable que dada la calidad, competencia, magnitud y trascendencia de cada uno de los programas enunciados, 
ninguno de ellos se orienta realmente a propiciar el desarrollo, observándose que hay en ellos más que la tendencia al cambio, la intención de mantener el deterioro conveniente para una comunidad como Tehueco.

Un 21\% (Figura 14) de la población entrevistada expresó que sí ha visto cambios positivos en la comunidad con la aplicación de estos programas, las oportunidades de que los niños y jóvenes continúen sus estudios por medio del Programa Oportunidades y la implantación de una Mercería por medio del Programa de Apoyo a los Proyectos de Inversión Rural (PAPIR), ya que, aseguran, ha contribuido al ahorro en la economía familiar pues hoy no tienen que ir tan lejos pagando pasajes para comprar útiles escolares, agujas, hilos, entre otras cosas necesarias en la familia.

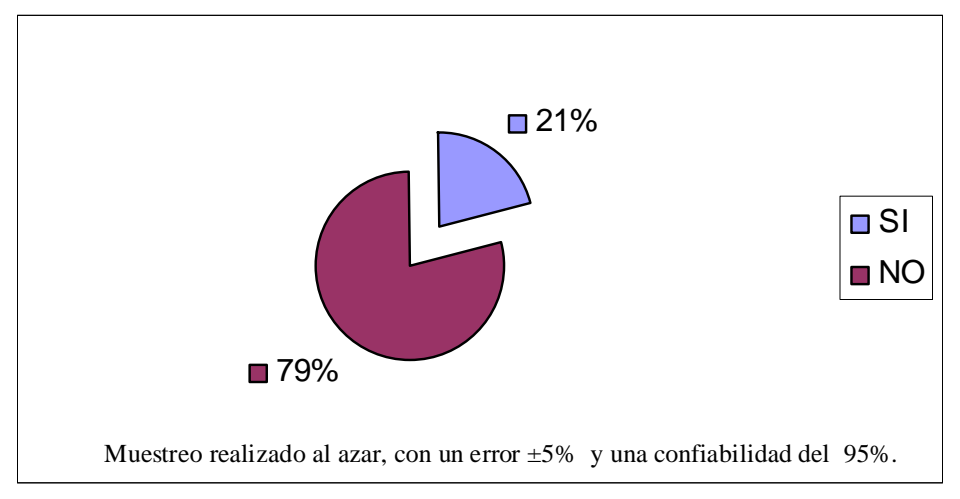

Figura 14. Cambios Positivos.

El resto de la población encuestada dijo que no se han visto cambios argumentando y reconociendo que lo antes señalado son sólo ayudas, que si bien es cierto contribuyen al gasto familiar, pero no generan ningún cambio ya que la comunidad sigue igual de atrasada que antes de la aplicación de estos programas.

La Figura 15 nos muestra cómo es que casi el $80 \%$ de la población piensa que los programas de gobierno dirigidos al desarrollo rural, que se han aplicado en la comunidad de Tehueco no han propiciado ningún tipo de desarrollo, sino han mantenido el estancamiento en la que se encuentra desde hace muchísimos años. 


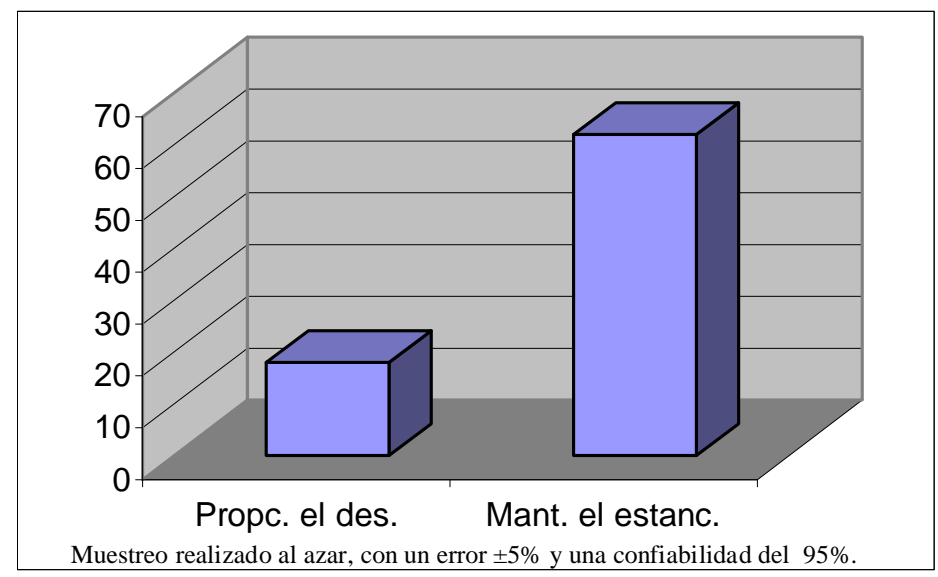

Figura 15. Acciones de los Programas.

Y por si fuera poco los habitantes de la comunidad señalan como principal responsable del atraso socioeconómico, en primer lugar al gobierno, por no proponer y ejecutar en la comunidad proyectos productivos viables a la solución de los problemas de la misma; que generen empleos y propicien un desarrollo sustentable aprovechando y explotando racionalmente los recursos naturales y humanos con los que cuenta la comunidad. En segundo lugar, a las autoridades de Tehueco por no ser capaces de solucionar los problemas que se viven y de forma contraria contribuyen en la agudización de éstos. Por último, a los mismos habitantes de la comunidad por no organizarse, por ser conformistas con lo poco que les dan, por la falta de compromiso y desinterés por mejorar su calidad de vida, como se muestra en la Figura 17.

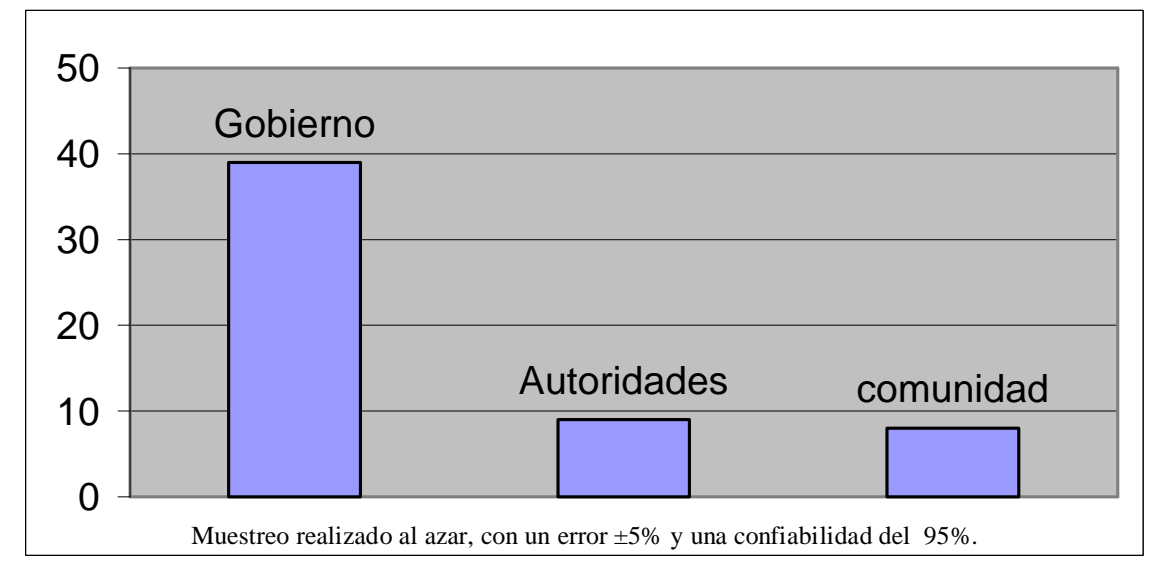

Figura 17. Responsables del Estancamiento. 
Es así que de las opiniones consensuadas en la comunidad, se observó, que todas estas políticas de atención al campo, implementadas en Tehueco, no pasan de ser compresas y medianías absolutamente intrascendentes para el cambio y el desarrollo.

\section{CONCLUSIONES}

Como conclusión, es importante mencionar que el desarrollo inducido en una comunidad es un proceso lento; en el que tanto los habitantes como el gobierno-por medio de las dependencias encargadas de hacer este proceso de desarrollo-, deben trabajar de manera conjunta, comprometida y responsable para lograr su propósito.

Estamos hablando aquí, que la atención gubernamental hacia este tipo de congregaciones de ninguna forma permite el albedrío comunitario ni sus acciones autónomas. Como lo menciona Ezequiel Ander-Egg al señalar que: “... el desarrollo de la comunidad no es una acción sobre la comunidad sino es una acción de la misma en la que necesariamente tienen que intervenir actores ajenos a ella”.

En el caso de la comunidad de Tehueco que fue nuestro objeto de estudio, este proceso de desarrollo ha sido demasiado lento-ha tardado cientos de años- ya que la comunidad tiene más de 500 años de fundación, y por mucho tiempo los habitantes habían tratado de lograr este desarrollo por su propia cuenta, no fue sino hasta hace poco que el gobierno empezó a intervenir en este proceso por medio de programas dirigidos a contribuir en el desarrollo por medio de diversas acciones, como la superación de la pobreza, el desarrollo de capacidades y el fomento del empleo, programas que actualmente están funcionando en la comunidad de Tehueco; con algunas deficiencias como la selección de los beneficiarios y la falta de información a toda la comunidad que es parte esencial para que la población confíe y esté dispuesta a participar en ellos. 


\section{LITERATURA CITADA}

Aguirre, M., T. Filesi y M. González.

2000 "Globalización y sistema internacional. Las claves para entender la realidad mundial".

D ICARIA, Centro de Investigaciones para la Paz. Barcelona. 80 p.

Ander-egg E.

1982 “Metodología y práctica del desarrollo de la comunidad".

El Ateneo, México, 342 p.

Atienza, J.

2000 "La Deuda externa y los pueblos del Sur. El perfil acreedor de España".

Caron, J Manos Unidas, Madrid. 135 p.

1996 “Una teoría ecológica para la intervención comunitaria: acceso y conservación de los recursos"

Revista de Intervención Psicosocial 2:14-23.

Entrena, $\mathrm{F}$.

1998 “Cambios en la construcción de lo rural. De la Autarquía a la Globalización"

Técnos, España. 56 p.

Gómez A. J.

1977 "Introducción al muestreo"

Tesis de Maestría en Ciencias. Colegio de Postgraduados. Texcoco, Estado de México. 247 p.

Herrera, T. F.

2004 "Los Paradigmas actuales del desarrollo rural en México"

Revista Académica de Economía 1:23-34.

López, T. R.

2004 "Despacho de Asesoría Integral Comunitario, Opción para el Desarrollo Sostenible de las Comunidades Rurales del Norte de Sinaloa" Tesis de Licenciatura. Universidad Autónoma Indígena de México. 106 p.

NACIONES UNIDAS

1987 "Nuestro Futuro Común: Informe Brundtland".

"Comisión Mundial del Medio Ambiente y del Desarrollo"

NACIONES UNIDAS

2000 “Objetivos de la Declaración del Milenio”. (En línea).

Disponible en www.un.org/millenniumgoals 
Quintero, A. M. A.

1997 "Tehueco, Tradiciones y Costumbres"

COBAES, México. 139 p.

Vargas, H. J. G.

2004 "Las Relaciones de los Fundamentos Teóricos entre la "Nueva Economía Política", la "Nueva Gerencia Publica" y la "Nueva Política Social"

Facultad de Economía. Universidad de Colima, México.89 p. 